\title{
Cancer is Easily Cured in a Clinic in Spain
}

\author{
Andrew Hague* \\ President of Cell Sonic Limited, Spain
}

*Corresponding author: Andrew Hague, President of Cell Sonic Limited, Spain.

\section{Abstract}

An independent report from a new CellSonic VIPP [1] user in Spain shows that cancer cells are changed to non-cancerous immediately they are treated. The implications for all cancer patients are profound.

\section{February 2020 Tuesday}

\section{Hello Andrew}

The CellSonic arrived and I quickly put it to work

Was easy to set up

I checked each patient again with the Bio Resonance test before treatment.

After treatment I tested each patient immediately.

Good News! The signal pointed to the LEFT before (showing cancer) then to the RIGHT immediately after the treatment (showing no cancer).

All my patients were amazed and so was I.

I also treated frozen shoulders [2], sciatica and a few other issues and it seems to have an immediate improvement.

I will be keeping careful records and sharing them with you.

\section{Friday}

We continue to get excellent results with cancer and other health concerns.

This has been a very good week and I am keeping good notes.

I have already used up 1 shockhead, so I need to order more.
I can for sure vouch for this machine.

When we run our Bio Reasonance tests now after the CellSonic treatments we are seeing a totally different result. We use German and American equipment to test our patients and we can see the presence of cancer and many other issues. Now we notice that after the treatment with the CellSonic the results show an incredible improvement. Also, it's very interesting. Instead of showing all kinds of dead cancer debris in the lymphatic system and so on we're seeing none of that. So, the cancer is not killed, but it's being converted into healthy cells with the electrical charge being restored. This puts no burden on the body and the patient feels much better. I am testing my patients a week after the treatment and the signal still points to the right showing that the electrical charge maintains itself and the cells are now functioning as healthy cells. This is absolutely outstanding.

\section{Discussion}

Dr Jenkins observations raise important points.

The electrical properties of cells are the key to curing cancer [3]. To stop the mutation of malignant cells, the electrical charge must be changed. This is done immediately with a CellSonic VIPP treatment.

No drugs that would kill cells are used so there are no side effects [4]. 
Ideally, a cancer patient will have had no previous treatment that harms their immune system. If a person with cancer decides to receive chemotherapy and/or radiation, they would have to wait 4 weeks afterwards to receive CellSonic therapy, as these potent drugs would interfere with the restoration of the electrical charge of the cells. Ideally someone with cancer would do the CellSonic therapy first.

Budwig Center often gets patients who have been failed by conventional treatments. Therefore, they need to do a lot of detoxification using different types of clays, herbs, coffee enemas, infrared saunas and rebounding to activate the lymphatic system. Emotional toxicity is another contributing factor to cancer and the Budwig Center uses four different emotional rebalancing techniques. In addition, they use natural supplements to boost the immune system.

Since I discovered the cure for cancer almost four years ago [5], CellSonic users have struggled to help patients whose cancers have brought them close to death. Patients who have recently succumbed to cancer are easy to restore to health. Dr Jenkins' measurements show that the cancer is converted immediately to healthy cells but that alone does not restore the rest of the injured body as quickly.

One of Dr Jenkins' specialities is a natural remedy that reinforces the immune system. In other words, for patients who have suffered at the hands of conventional cancer treatments, stopping their cancer is only the first stage in recovery. The more difficult task is to recover from the inflicted injuries.

The sooner a patient asks for CellSonic treatment, the better. The CellSonic cancer cure is widely published and readily available [6].

In the USA, it is believed that the cost of care and treatments for a cancer patient amounts to $\$ 200,000$ a year and they die in the fifth year. The Budwig Center has a mission of helping as many people with cancer as possible and their fees very affordable at $€ 5000$ per week.

CellSonic treatment can be done in half an hour or less. Many users have been astounded by its simplicity saying that it could be done at the roadside with the CellSonic machine on battery power. This may eventually happen as it becomes available in poorer parts of the world where cancer is as prevalent as it is in rich countries.

The CellSonic VIPP technique adjusts to all cancers. It has been used for forty years since kidney stones were first pulverised by sound waves with millions of patients safely treated in all countries. It was this assurance of safety that allowed me to proceed with the first cancer treatment. No drugs have been tried for as long on as many patients with a zero-harm result.

\section{Cost Saving}

The cost saving from CellSonic is huge. For example, in Britain with a population of 65 million, the expenditure on cancer is about $£ 15$ billion ( $\$ 19.5$ billion) a year. Wound healing amounts to $£ 5$ billion a year. Lower back pain and similar aches and pains incur cost that may be more especially when including the days off work. With cancer curable in minutes and diabetic non-healing ulcers healed, the financial burdens on governments, insurance companies and their clients are reduced to a few hundred dollars a year for each person and that's before thinking about removing the pain and suffering.

\section{Conclusion}

What was done last week at Budwig Center can be done anywhere. Dr Jenkins has the complete package, not just CellSonic. His contact details are given above and he welcomes enquiries for his knowledge and advice. Many doctors who accept that they are very limited with the tools they are currently using to cure cancer can now access an existing system and do for themselves what $\mathrm{Dr}$ Jenkins did. His discovery is displayed above, and this knowledge will spread as many sincere doctors and practitioners look for a totally natural but highly effective cancer treatment program, like the Budwig protocol in conjunction with CellSonic therapy.

\section{Acknowledgement}

None.

\section{Conflict of Interest}

Author Declare no conflict of interest.
References
1 Cancer. CellSonic.
2 Movement. CellSonic.
3 Steve Haltiwanger. The Electrical Properties of Cancer Cells.
4 Cancer the ultimate plan.
5 Cancer can be Cured.
6 Vitamed centrum medyczne. 